\title{
Uncertainty and precaution in European environmental risk assessment of chemicals
}

\author{
F.A.M. Verdonck*,** P.A. Van Sprang ${ }^{* *}$ and P.A. Vanrolleghem* \\ *Department of Applied Mathematics, Biometrics and Process Control (BIOMATH), Ghent University, Gent, \\ Belgium (E-mail: frederik.verdonck@biomath.ugent.be) \\ **EURAS, Rijvisschestraat 118 box 3, B-9052 Gent-Zwijnaarde, Belgium \\ (E-mail: patrick.vansprang@euras.be, peter.vanrolleghem@biomath.ugent.be)
}

\begin{abstract}
It is recognised that there is a need for a proper treatment and transparency of uncertainty in risk assessment and management, especially in view of the upcoming proposed new chemical policy REACH, which delegates the responsibility for conducting risk assessments to industry. The current EU risk assessment for new and existing substances is largely deterministic and prudential measures are implicitly embedded in calculation schemes and rules. In this paper, a more probabilistic approach to risk assessment is advocated. The advantage is twofold: 1) inherent variability and other uncertainty pertaining to exposure and effects are transparently taken into account, while at the same time 2) issues of caution are explicitly transferred to the risk management phase. The result of a probabilistic risk assessment as suggested is improved transparency with quantitative and qualitative uncertainty estimates. Such uncertainty information can be used to discuss precautionary measures in the context of risk management.

Keywords Precaution; probabilistic risk assessment; technical guidance document; uncertainty
\end{abstract}

\section{Introduction}

Environmental pollution of toxic substances has led to regulations on the production and use of chemicals. Often the tools used for assessing the potential impact of chemicals to ecosystems are risk-based. In general, such risk analysis process can be subdivided into three main phases, i.e. risk assessment (hazard identification, exposure and effects analysis, risk characterisation), risk management (process used for deciding between policy alternatives in consultation with stakeholders e.g. mitigation, cost-benefit, etc.) and risk communication (risk terminology according to EC (2000)). In practice, risk analysis is a continuous iterating process between these sometimes undistinguishable phases. All phases are needed to control adequately the potential risks of chemicals to the environment, but there is a need for functional separation of risk assessment and risk management, to ensure the independence of risk assessment. This was one of the key conclusions of a recent European workshop on "improving the interface between risk assessment and risk management" (Hart, 2003). However, efficient interaction between risk assessors and risk managers is essential; to ensure that risk assessment is relevant to the needs of risk management and to optimise regulatory decision-making.

The European Commission (EC, 2000) describes a controversy as to the role of scientific uncertainty in risk analysis, and notably as to whether it belongs under risk assessment or risk management. According to EC (2000), this controversy springs from a confusion between a prudential approach and application of the Precautionary Principle (PP). These two aspects are according to EC (2000) complementary but should not be confounded. The prudential approach is part of risk assessment policy which is determined before any risk assessment takes place and is therefore an integral part of the scientific opinion delivered by the risk evaluators. On the other hand, application of the PP is part of risk management, when scientific uncertainty precludes a full assessment of 
the risk and when decision-makers consider that the chosen level of environmental protection or of human, animal and plant health may be in jeopardy (EC, 2000).

The main goal of environmental risk assessment of new and existing substances is to answer the question: "What is the likelihood (i.e. probability) of adverse effects occurring to exposed ecological systems due to exceedance of a toxicity level by an environmental concentration?" However, the current risk assessment tries to answer the question: "Is it likely that adverse effects occur to exposed ecological systems due to exceedance of a no effect level by an environmental concentration?" It is based on the comparison of a Predicted Environmental Concentration (PEC) with a 'Predicted No Effect Concentration' (PNEC). The PNEC should be protective for a specific environmental compartment and is based on a set of (acute or chronic) toxicity test results (i.e. testing species sensitivity) (EC, 1996 and 2003). Prudential aspects (such as worst-case assumptions) or adopting an assessment factor (also named safety factor as in EC (2000) or uncertainty factor as in Sanderson et al. (2005)) to account for extrapolation uncertainty (the magnitude of this factor depends on the degree of extrapolation uncertainty of the available data, typically 1-1000) are incorporated in the risk assessment procedure (EC, 2000; EC, 2003).

Usually following the risk assessment phase, risk management is a process used for deciding between policy alternatives in consultation with stakeholders (interested parties), taking account of both risk and its uncertainty (based on a scientific evaluation as complete as possible) and other factors (e.g. social, economic, and legal considerations) and, if required, selecting and implementing appropriate control options. The scientific evaluation should identify the degree of scientific uncertainty, and decision-makers should take those uncertainties, among others, into account when determining whether to trigger action based on the PP (Hart, 2003).

However, some prudential measures in risk assessment (such as assessment factors) can be subjective (when not properly defined or not possible to define in guidelines). In addition, the uncertainty and variability should be quantified if the data are available. Until now, these prudential measures in the in-depth risk assessment may not entirely be considered as inappropriate mainly because the EU member states themselves are conducting both risk assessment and management and consequently both phases are to some extent integrated. But the new chemical policy REACH for Registration, Evaluation, Authorization of Chemicals (CEC, 2003) is proposing to delegate the responsibility of data compilation, risk assessment and risk reduction of chemicals from the authorities to industry.

The goal of this paper is therefore to explore how some prudential measures can be transferred from risk assessment to risk management (Figure 1). For this, it is investigated how uncertainty and related prudential measures are considered in the deterministic, the probabilistic and the current EU risk assessment, which is to a large extent deterministic. The ongoing Zn EU risk assessment will be used as an example throughout the paper.

\section{Deterministic environmental risk assessment}

The fundamental principles and methodology for European risk assessment of new notified and existing substances under commission directive 93/67/EEC and commission regulation (EC) No 1488/94 is described in the EU Technical Guidance Document (TGD) and is implemented in the computer program EUSES (European Union System for the Evaluation of Substances) (EC, 1996). This was designed to be a decision-support system for the evaluation of the risks of substances posed to man and the environment. The EU-TGD can be considered as a kind of consensus document developed and extensively discussed with experts from member states, NGOs and industry (Bodar et al., 2003) to conduct comprehensive chemical risk assessments. 


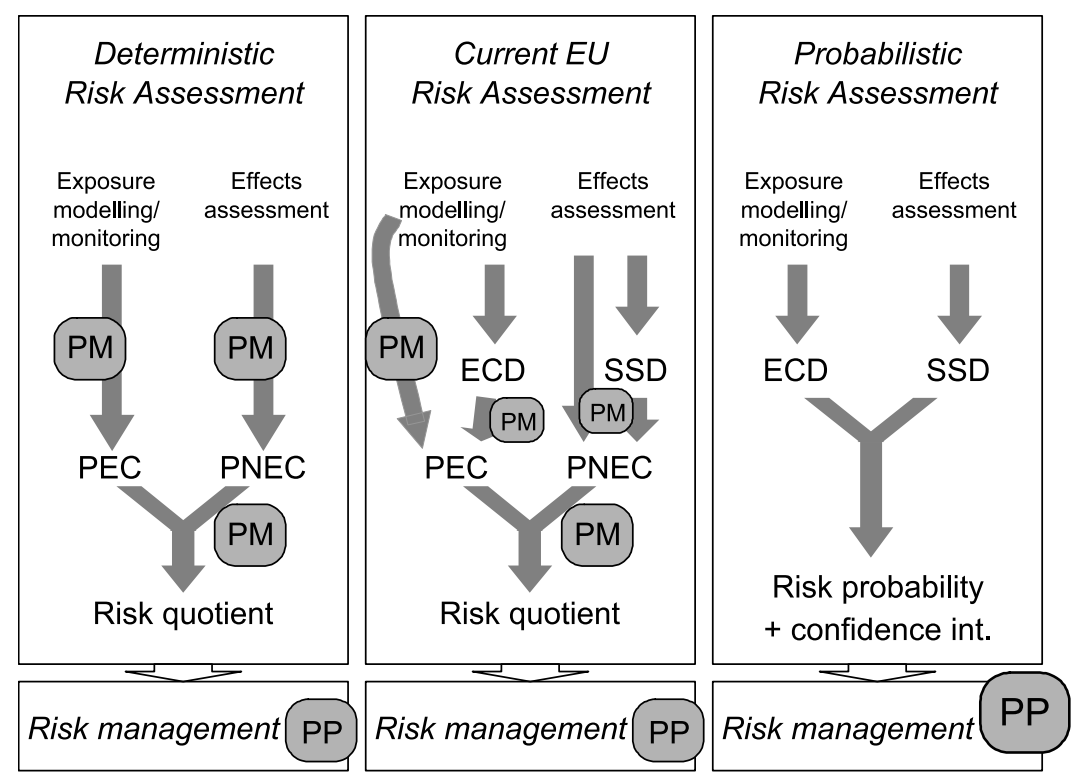

Figure 1 Overview of the implementation of the Prudential Measures (PM) and the Precautionary Principle $(\mathrm{PP})$ in the deterministic, probabilistic and current EU risk assessment and management

Having conducted the exposure and effects assessments for all environmental compartments, a quantitative risk characterisation is carried out. This consists of comparing the concentrations at which no effects are expected (i.e. the PNEC) with the estimated exposure level (i.e. the PEC) for the considered chemical. In this deterministic framework, a risk quotient, PEC/PNEC ratio (see Figure 1 left), larger or equal to one signifies that there is a potential risk of adverse effects occurring and a large quotient is likely to indicate a high level of risk. A risk quotient smaller than one signifies no risk (considering the conservative assumptions made during the assessments). The key question in risk assessment is therefore answered by the deterministic risk characterisations with a "yes, there is potential risk / no risk" statement.

A general rule for predicting the environmental concentration is that the best and most realistic information available should be given preference. However, it may often be useful (e.g. in the absence of sufficiently detailed data) to initially conduct an exposure assessment based on worst-case assumptions, and using default, conservative values when model calculations are applied. In case the risk characterisation based on worstcase assumptions for the exposure indicate that the substance is "of no concern," the risk assessment for that substance can be stopped with regard to the compartment considered. If, in contrast, the outcome indicates that a substance is "of concern", the assessment must, if possible, be refined using a more realistic exposure prediction.

In current environmental risk assessments, uncertainties are not explicitly quantified. They are only arbitrarily considered in assessment factors or worst-case assumptions and are only applied in the exposure, effects and risk characterisation as a (pre)cautionary measure. Some examples demonstrating how prudential measures are implicitly embedded in calculation schemes and rules of risk assessment are discussed below.

In the exposure assessment, PECs are usually calculated based on production or processing tonnages and default release fractions. A limited survey carried out by Jager (1998) concluded that these TGD-defaults are generally higher, i.e. more conservative, than the site-specific release factors. In some cases the difference between site-specific and default release factors is up to three, in extreme cases up to four, orders of 
magnitude. In other cases, the default values are quite accurate. The current TGD-defaults for emission release estimation can thus be considered as prudential estimates, which often result in quite conservative exposure calculations.

For most substances in the effects assessment, the pool of data from which to predict ecosystem effects is often very limited as, in general, only short-term toxicity data are available (EC, 1996). In these circumstances, it is recognised that, while not having a strong scientific validity, pragmatically derived assessment factors must be used. In applying such factors, the intention is to predict a concentration below which an unacceptable effect at ecosystem level will most likely not occur. In establishing the size of these assessment factors, a number of uncertainties are addressed to extrapolate from singlespecies laboratory data to a multi-species ecosystem. These may best be summarised under the following headings (EC, 1996):

- intra- and inter-laboratory variation of toxicity data;

- intra- and inter-species variations (biological variance);

- short-term to long-term toxicity extrapolation;

- laboratory data to field impact extrapolation (additive, synergistic and antagonistic effects from the presence of other substances may also play a role here).

The size of the assessment factor depends on the confidence with which a PNEC can be derived from the available effects data. This confidence increases if data are available on the toxicity to organisms at a number of trophic levels, taxonomic groups, and with life histories representing various feeding strategies. In practice, an assessment factor between 10 and 1,000 is applied on the lowest toxicity level of the relevant available toxicity data. Prudential measures are thus already embedded in the PNEC derivation. The subsequent risk quotient (defined as the ratio of PEC and PNEC) will consequently also be a worstcase estimate which moreover does not contain any uncertainty quantification.

This deterministic risk assessment approach is not realistic because PEC and PNEC are considered as single, crisp values whereas in reality they are characterised by both uncertainty and variability. Accounting for this would avoid problems associated with using worst-case assumptions (e.g. lack of consensus in defining the worst-case, and the generation of unrealistically extreme assessments by combining multiple worst-case assumptions). The methods also do not encourage further research. In the deterministic framework, risk is not expressed as a probability (as it should be, see general risk definition in introduction). Instead, risk is expressed as a ratio, basically a "yes/no risk"-statement. And the degree of conservatism is unknown. Thus, it is not clear whether the risk quotient represents a reasonable worst case or an unrealistic, "over-protective" situation. This has been criticised in literature (e.g. Jager et al., 2001a). In short, the conventional methods make use of conservative and insufficiently transparent, realistic, scientific assumptions. However, these conventional methods are likely to remain the primary tool for lower tiers of risk assessment because they are simple and rapid, and are appropriate for use as screening tools provided they are sufficiently conservative (i.e. over-protective). The subsequent risk management may additionally apply measures based on the PP.

\section{Probabilistic environmental risk assessment}

The drawbacks of the deterministic ecological risk assessment have therefore led to the development of more probabilistic or quantitative uncertainty analysis techniques in this area, of which the importance and usefulness is often stressed in literature (e.g. Cullen and Frey, 1999; Jager et al., 2001b; Verdonck, 2003). For data-rich substances, where acceptable ranges of toxicity/exposure data are available, a Probabilistic Environmental 
For data-poor substances, the degree of conservatism due to uncertainty introduced at each level of the risk assessment process can be estimated by conducting a deterministic risk assessment for the following two scenarios: one with realistic worst-case assumptions and one without the build up of worst case assumptions or assessment factors. The difference in risk quotients between the realistic worst-case and typical scenario can thus be considered as a quantitative estimation of the total level of conservatism.

In PERA for data-rich substances, the exposure concentration and species sensitivity are treated as random variables taken from probability distributions (respectively Exposure Concentration Distribution (ECD) and Species Sensitivity Distribution (SSD)) which are combined to give a risk probability (see Figure 1 right and visualised in Figure 2 in the PERA of $\mathrm{Zn}$ in the Dutch surface water (Van Sprang et al., 2004)). In these probabilistic types of environmental (and human) risk assessments, the distinction between data uncertainty and variability should be made (e.g. Hoffman and Hammonds, 1994; Cullen and Frey, 1999). Variability represents inherent heterogeneity or diversity in a well characterised population. Fundamentally a property of nature, variability is not reducible through further measurement or study. At the exposure side, the temporal and spatial variations of chemical concentrations can be captured in a variability distribution, called ECD. At the effects side, various species sensitivities towards a chemical can also be captured in a variability distribution called SSD. Figure 2 shows the ECD and SSD variability distributions as a black line for $\mathrm{Zn}$ in the Dutch surface water (from Van Sprang et al., 2004). Uncertainty can be visualised as confidence intervals. In Figure 2, the uncertainty is shown as a band around the cumulative variability distribution function for $\mathrm{Zn}$ in the Dutch surface water. For each percentile of the variability distribution, an uncertainty or confidence interval can be calculated (i.e. the uncertainty distribution). Uncertainty represents partial ignorance or lack of perfect information about poorly characterised phenomena or models, and can partly be reduced through further research (Cullen and Frey, 1999). There are many sources of uncertainty, e.g. sampling uncertainty, measurement error, model structure uncertainty ... (Cullen and Frey, 1999).

The key question (i.e. "What is the likelihood (i.e. probability) of adverse effects occurring to exposed ecological systems due to exceedance of a toxicity level by an environmental concentration?") in PERA is then answered with a risk probability and an uncertainty or confidence interval. In this way, both the inherent variability and uncertainty of the environmental concentration and the species sensitivity are explicitly accounted for and not hidden in calculation schemes and rules. PERA therefore delivers a more transparent and realistic approach to estimate risks. In the example for $\mathrm{Zn}$, the

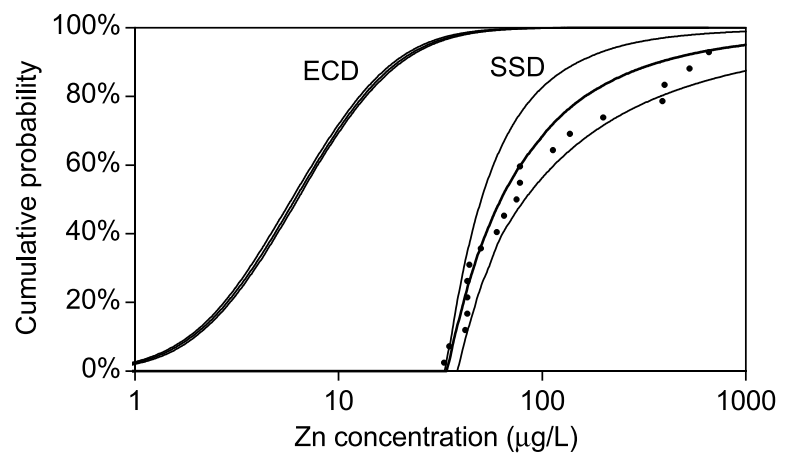

Figure 2 Probabilistic Environmental Risk Assessment (PERA) with Exposure Concentration Distribution (ECD) (left) and Species Sensitivity Distribution (SSD) (right), each with $90 \%$ uncertainty bands (from Van Sprang et al., 2004) 
probability that the environmental concentration in Dutch surface waters exceeds the species sensitivity is $0.5 \%$. The estimated $90 \%$-confidence interval is $0.1-0.9 \%$.

In PERA, worst-case assumptions or assessment factors inspired by issues of caution are postponed where possible. The uncertainty and variability is quantified as much as possible resulting in a realistic risk probability estimate with uncertainty or confidence interval. However, PERA is not yet able to capture all sources of uncertainty. In the aforementioned example, only parameter or sampling uncertainty was considered. The remaining, unquantifiable uncertainty can be added as qualitative statements. For example, laboratory to field extrapolation uncertainty, choice of Log normal and Pareto probability distribution, and model uncertainty, etc., are difficult to quantify. Moreover, ecologically relevant numbers of species needed to develop the SSD including species with variable lifehistories and role in the ecosystem (e.g. keystone species) are not defined in terms of incorporation requirements for SSDs. Also the SSDs cannot integrate secondary effects of perturbation and functional impacts, and can thus not replace the highest tier of ecological risk assessment (mesocosm). Once this information is clearly communicated to risk managers or decision-makers, those uncertainties should be taken into account when determining whether to trigger action based on the PP and other factors (e.g. social, economic and legal considerations) in the risk management phase.

\section{Current EU risk assessment}

In 2003, the EU-TGD was updated. The revised EU risk assessment methodology remains intrinsically a deterministic assessment but some probabilistic approaches were introduced or described in more detail (EC, 2003). The middle box of Figure 1 shows the current status of an EU risk assessment. Some examples are given in which more realistic approaches are used whereafter prudential measures are taken.

For example, if a large data set from long-term tests (at least 10 NOECs (preferably more than 15) for different species covering at least 8 taxonomic groups), SSDs may be used to derive a threshold (typically the 5th percentile of the SSD) in the effect assessment (EC, 2003). This threshold is then used to determine a PNEC. Although a threshold, derived from the SSD, is used in the risk characterisation instead of the SSD 'as such', there is an incentive to more realistic, probabilistic approaches. However, there is still a tendency to keep prudential measures in risk assessment. For final PNEC setting, an assessment factor between 1 and 5, reflecting the further uncertainties identified, needs to be applied on the derived threshold. As a minimum, the following points have to be considered when determining the size of the assessment factor (EC, 2003):

- the overall quality of the database and the endpoints covered, e.g. if all the data are generated from "true" chronic studies (e.g. covering all sensitive life stages);

- the diversity and representativity of the taxonomic groups covered by the database, and the extent to which differences in the life forms, feeding strategies and trophic levels of the organisms are represented;

- knowledge on presumed mode of action of the chemical (covering also long-term exposure);

- sampling uncertainty and probability distribution model structure uncertainty;

- comparisons between field and mesocosm studies.

In the ongoing $\mathrm{Zn}$ EU risk assessment, the rapporteur identified two issues that would support the use of an assessment factor higher than 1 for the derivation of the final PNEC value for the freshwater environment. From the in-depth analysis, it seemed that the results of laboratory and semi-field (model ecosystems) studies with zinc showed effects on biomass-related endpoints below the 5th percentile value for $\mathrm{Zn}$. The second argument was based on the observation that the 5th percentile value for $\mathrm{Zn}$ (implying a 95\% 
protection level) may not be sufficiently protective, as individual toxicity values for different species were below the estimated threshold value for $\mathrm{Zn}$.

Large uncertainties (like the ones described above), which may rise to (pre)cautionary measures, usually lead to further scientific research and further research leads to a more realistic risk (whether increased or decreased) (EC, 2000). However, further research can also lead to an increased or decreased uncertainty. In case of increased uncertainty, the saying "the more we know, the more we know we don't know" is applicable. Unlike in the probabilistic risk assessment, risk and uncertainty are not treated separately in the EU risk assessment. Consequently, increasing uncertainty leads automatically to an increased risk in the current EU risk assessment whereas in the probabilistic risk assessment, increasing uncertainty leads to an increased uncertainty interval around the probabilistic risk irrespective of an increasing or decreasing risk. Issues of (pre)caution should therefore be avoided prior to risk characterisation by explicitly considering the uncertainties (preferably quantitative, if not possible qualitative). Such uncertainty information can be used to discuss precautionary measures in the context of risk management.

\section{Conclusions}

Risk assessment is the science-based process used to identify and characterise hazards, exposure, risk, and characterise its uncertainty. Risk management is the decision-making process used for deciding between policy alternatives in consultation with stakeholders, taking into account both the estimated risk and its uncertainty, the precautionary principle, the cost and other factors (e.g. social, economic and legal considerations). There is a need for a proper treatment and transparency of uncertainty in risk assessment of new and existing substances. This is important in the existing EU chemical risk assessment procedures, but even more in view of the upcoming proposed new chemical policy REACH, which delegates the responsibility for conducting risk assessments to industry. The current EU risk assessment for new and existing substances is largely deterministic and prudential measures are implicitly embedded in calculation schemes and rules. Probabilistic techniques in environmental risk assessment are more precautionary-driven because they take into account the inherent variability and uncertainty of exposure and effects and result therefore in a more relevant probabilistic risk with both quantitative and qualitative uncertainty estimates. In this way, issues of caution (prudential measures) are explicitly transferred to the precautionary principle analysis in the risk management phase.

\section{Acknowledgements}

The authors would like to thank Dr. Van Asselt (Maastricht University) and two anonymous reviewers for their useful suggestions. This research was funded by a scholarship from the Flemish Institute for the Improvement of Scientific-Technological Research in the Industry (IWT) and by the EU project Harmoni-CA (Contract no: EVK1-200100192).

\section{References}

Bodar, C., Berthault, F., de Bruijn, J., van Leeuwen, C., Pronk, M. and Vermeire, T. (2003). Evaluation of EU risk assessments existing chemicals (EC Regulation 793/93). Chemosphere, 53(8), 1039-1047.

CEC (2003). Proposal for a regulation of the European Parliament and of the Council concerning the Registration, Evaluation, Authorisation and Restrictions of Chemicals (REACH) establishing a European Chemical Agency and amending Directive 1999/45/EC and Regulation (EC) \{on Persistent Organic Pollutants \}. In COM, 2003, 644.

Cullen, A. and Frey, H. (1999). Probabilistic techniques in exposure assessment. A handbook for dealing with variability and uncertainty in models and inputs. Plenum. New York. 
EC (European Commission) (1996). Technical guidance document in support of commission directive 93/67/ EEC on risk assessment for new notified substances and commission regulation (EC) $\mathrm{N}^{\circ} 1488 / 94$ on risk assessment for existing substances and directive 98/8/EC of the European parliament and of the council concerning the placing of biocidal products on the market. Commission of the European Communities. Brussels, Belgium.

EC (European Commission) (2000). Communication from the commission on the precautionary principle. COM, 1.

EC (European Commission) (2003). Revised EU technical guidance document, IHCP/JRC. Ispra, Italy.

Hart, A. (2003). Improving the interface between risk assessment and risk management, Report of European workshop, 3-5 September 2003, Central Science Laboratory, Sand Hutton, UK.

Hoffman, F. and Hammonds, F. (1994). Propagation of uncertainty in risk assessments: the need to distinguish between uncertainty due to lack of knowledge and uncertainty due to variability. Risk Analysis, 14(5), 707-712.

Jager, T. (ed.) (1998). Evaluation of EUSES: inventory of experiences and validation activities. RIVM, Report no. 679102048 Bilthoven, The Netherlands.

Jager, T., den Hollander, H., van der Poel, P., Rikken, M. and Vermeire, T. (2001a). Probabilistic environmental risk assessment for dibutylphthalate (DBP). Human and Ecological Risk Assessment, 7(6), $1681-1697$.

Jager, T., Vermeire, T., Rikken, M. and van der Poel, P. (2001b). Opportunities for a probabilistic risk assessment of chemicals in the European Union. Chemosphere, 43(2), 257-264.

Sanderson, H., Stahl, C., Irwin, R. and Rogers, M. (2005). Reflections on uncertainty in risk assessment. Wat. Sci. Tech., 52(6), 73-80.

Van Sprang, P., Verdonck, F., Vangheluwe, M., Vanrolleghem, P. and Janssen, C. (2004). Probabilistic environmental risk assessment of zinc in Dutch surface waters. Environmental Toxicology and Chemistry, 23(12), 2993-3003.

Verdonck, F. (2003). Geo-referenced probabilistic ecological risk assessment. PhD. Thesis, Faculty of Bioscience Engineering, Ghent University. 